\title{
An examination of the nonspecific skin resistance response
}

\author{
DANIEL M. BAUGHER \\ Rutgers-The State University, New Brunswick, New Jersey 08903
}

\begin{abstract}
Various aspects of the nonspecific skin resistance response were investigated. Nonspecific activity was found to be directly related to subjective reports of arousal and performance in a vigilance task, indicating that it may be a useful measure of arousal. In addition, the stability of nonspecific activity was found to be relatively high across similar experimental conditions but was rather low across dissimilar conditions, suggesting that this activity does not possess any strong "trait-like" characteristics.
\end{abstract}

Research which uses measures of skin resistance as a dependent variable is increasing as the apparatus for monitoring skin resistance becomes more available and the procedure for recording and scoring become standardized (see Lykken and Venables, 1971). Many aspects of continuously recorded skin resistance can be scored; this paper is concerned with nonspecific responses, rapid changes in skin resistance which occur in the apparent absence of external stimulation.

The psychological significance of nonspecific skin resistance responses is controversial. According to Lacey and Lacey (1958) these responses are not components of emotion but are, rather, a sort of "biological noise coming from the physiochemical processes at the level of the localized cell aggregates" (p. 163) and, consequently, are "psychologically silent." An alternate viewpoint is represented by the research of Zuidema, Cohen, Silverman, and Riley (1956), who found that the frequency of nonspecific responses increased significantly as a function of increased stress in their subjects and McDonald, Johnson, and Hord (1964), who showed that alert subjects produced more nonspecifics than drowsy subjects in similar experimental conditions. These studies suggest that nonspecific skin resistance responses are related to level of arousal and, as such, do relay some information regarding an individual's psychological functioning.

The purpose of this study is to examine three aspects of the nonspecific skin resistance response: (1) the psychometric properties of reliability of the response from one time to another and the generalizability of the response from one setting to another, (2) the association between the person's self-report of arousal and skin resistance activity, and (3) the relationship between

A portion of this paper was presented at the meeting of the Eastern Psy chological Association, Washington, D. C., May 1973. Requests for reprints should be addressed to the author, Department of Psychology, Rutgers University, New Brunswick, New Jersey 08903 . All correlations are Spearman rank-order correlations reflecting Siegel's correction for a large number of tied ranks (Siegel, 1956, pp. 206-210). Professor John Gormly sponsors this paper and takes full editorial responsibility for its content. performance in a reaction time condition and skin resistance activity.

\section{METHOD}

\section{Subjects}

Subjects were 34 male students at the University of Virginia.

\section{Apparatus}

Palmar skin resistance was recorded on a Sanborn 150 heat pen recorder through a wheatstone bridge circuit, with an excitation voltage of $4.5 \mathrm{~V} \mathrm{dc}$ and sufficient internal resistance to provide an average current of 70 microamperes through each subject. Zinc-zinc sulphate electrodes were used with the effective area of the electrodes controlled $(3.18 \mathrm{~cm}$ in diam, spaced $.79 \mathrm{~cm}$ apart).

\section{Procedure}

The skin resistance of the subjects was examined during two separate experimental sessions with a hydration period of $10 \mathrm{~min}$ provided before each. On the 1 st day, the subject was placed in a barber chair in a reclining position in a dimly lit room and told to keep his eyes closed and to rest. White noise was administered throughout both sessions. The experimenter monitored the subject's skin resistance for a 15 -min period. Following this, the subject was asked to rate his stress and the emotional content of his thoughts during the session on a 1-7 scale. He was then told to report back the next day at the same time for a similar examination.

The second session contained a 15-min resting session identical in procedure to Session 1. After this, the subject was advised that a 40-min task session had begun and that he should remain quietly resting in the chair with his eyes closed. During this $40-\mathrm{min}$ period, a bright light came on five times, in varied intervals $(5,11,14,8$, and $2 \mathrm{~min})$ to which the subject was to respond as quickly as he could by pressing a button on the right side of his chair, which turned the light out. The light was clearly visible to all subjects through closed eyelids. After this session, the subject was asked to give two separate ratings of his stress and emotional cognition: one for the 15-min sessions (rest session) and one for the 40-min presentation session (task session).

\section{Basic Measure}

Skin resistance changes over both sessions were scored as follows. Each record was divided into 15-sec intervals with an interval scored as having a nonspecific resistance if a response of at least $2 \%$ of the present basal resistance setting occurred. Each interval was scored only once, even though it could possibly have more than one response within it. In addition, all intervals containing "known" specific responses were not counted. 


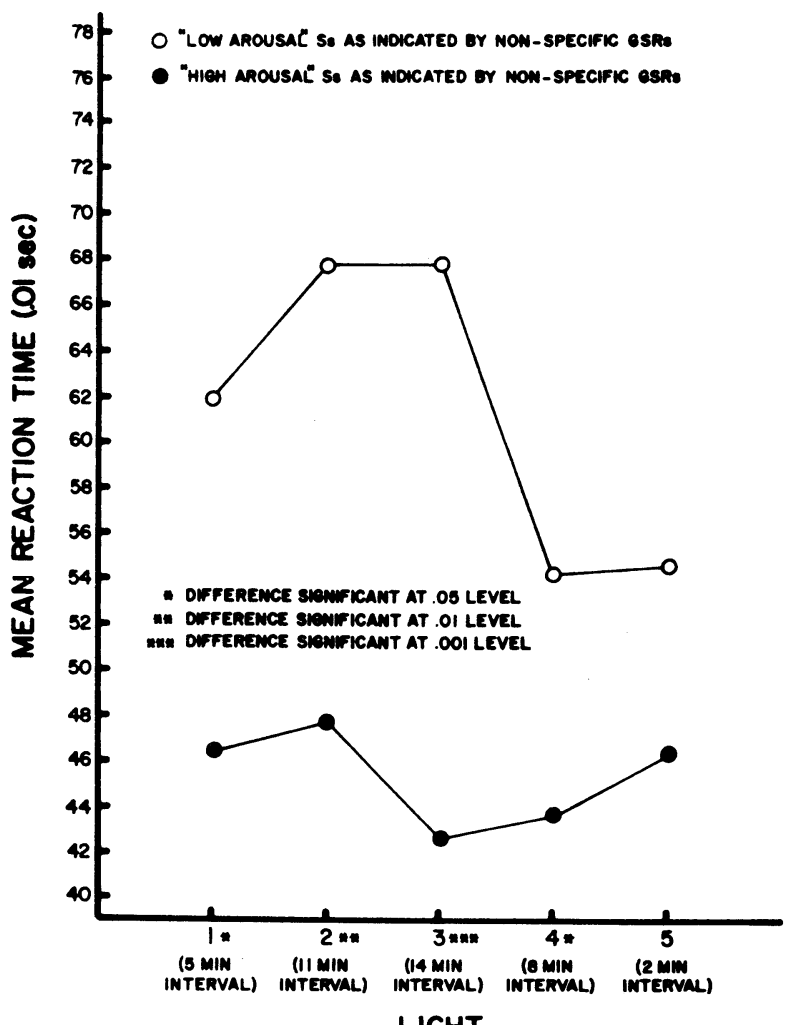

LIGHT

Figure 1. Nonspecific resistance response activity and performance.

Reaction time was recorded on a Lafayette timer to $.01 \mathrm{sec}$ for all five light presentations.

The measures of stress and emotional content were direct questions which asked the subject to make an interpretation of his feelings during each session on a scale of 1-7 where 1 represented high emotional content or stress, and 7 represented low emotional content or stress.

\section{RESULTS}

\section{Psychometric Properties}

Nonspecific resistance responses when analyzed across two similar experimental conditions, the first rest session and second rest session, show reliable individual differences ( rho $=.73, \mathrm{df}=34$ ). When examined across dissimilar conditions, the first and second rest sessions in contrast to the first $16 \mathrm{~min}$ of the task session, the resistance activity is not as stable and, moreover, seems to provide inconsistent results, with only the comparison of Rest Session 1 and the task session reaching significance (rho $=.35$ and .32 for the comparison between Rest Session 1 and the task session and Rest Session 2 and the task session, respectively; $d f=34$ ).

\section{Self-Report of Arousal}

Rank order correlations indicate a direct relation between the stress of the experimental sessions as rated by the subject and nonspecific resistance activity (rho $=.63, .48$, and .46 for Rest Session 1, Rest Session 2, and task session, respectively; $\mathrm{df}=34$ ). Rank order correlations also indicate a direct relation between subject ratings of emotional cognition during the sessions and nonspecific activity (rho $=.66, .72$, and .40 for Rest Session 1, Rest Session 2, and the task session, respectively; $d f=34)$. The subjective ratings were moderately stable across the two resting sessions (rho $=$ .53 and .63 for ratings of stress and ratings of emotional content, respectively; $d f=34$ ) but only the ratings of stress showed significant correlation $(p<.02)$ across the dissimilar conditions of the resting sessions and the task session (rho $=.43$ and .47 for the comparison between Rest Session 1 and the task session and Rest Session 2 and the task session, respectively; $\mathrm{df}=34$ ). In addition, the ratings were moderately intercorrelated across the experimental sessions (rho $=.37, .54$, and .62 for Rest Session 1, Rest Session 2, and the task session, respectively; $\mathrm{df}=34$ ).

Furthermore, both subjective ratings of arousal and nonspecific activity showed reliable shifts from the periods of rest to the task session. Sixty per cent of the subjects showed increases in their emotional cognition, feelings of stress, and nonspecific activity from Rest Session 1 to the task session and 56\% showed increases in all three measures from Rest Session 2 to the task session.

\section{Reaction-Time Performance}

Nonspecific resistance activity was predictive in the simple vigilance task as indicated in Figure 1. Subjects were classified according to the number of nonspecific responses they had before each light presentation by a split-median method into "high" and "low" arousal groups, with subjects able to change their status before each. "High" arousal subjects showed significantly quicker reactions in four out of five light presentations than did "low" arousal subjects.

\section{DISCUSSION}

Within the present study, nonspecific resistance activity was a stable measure across similar conditions. Its consistency across dissimilar conditions was, however, rather low, suggesting that it does not possess any strong "trait-like" characteristics. Of course, questions regarding a measure's reliability and generalizability are related to rather arbitrary distinctions concerning the number of "universes" across which a measure is expected to maintain consistency. Certainly, if nonspecific activity is considered to be a measure of arousal, it is not necessary to predict consistency or generalizability across various situations or "universes." Individuals are not necessarily aroused by the same things in different situations and, in turn, may change their rank order across these situations. Moreover, such inconsistency places some doubt upon the formulation of nonspecific responses as indicators of "biological noise" (Lacey \& Lacey, 1958). Within this framework, nonspecific activity should be rather consistent across different situations since a large proportion of its variance should be related to stable individual differences at a physiological level. 
The rather strong relationship found between subjective ratings of arousal and nonspecific activity in the present study indicates that this measure may be very useful in assessing individual differences in feelings of excitement or stress during a variety of situations. Further consideration also suggests that some nonspecific resistance responses may be linked to specific internal cues. It would seem likely that a person who rates himself as having emotionally "charged" thoughts would have specific responses to particular aspects of these thoughts. This is not to suggest, however, that all nonspecific resistance responses are covert specific responses since ratings of emotional cognition were only moderately redundant of nonspecific activity and the subjective measures, themselves, were only moderately related.

The direct relationship found between nonspecific activity and performance was expected, given the range of arousal elicited by the performance task. Subjects classified as having "high" arousal were probably only moderately aroused in relation to the possible range of arousal, since the task session was not designed to be stressful and, in fact, was rated by only one subject as highly stressing. More specifically, this outcome is predicted by the "arousal continuum," suggested by Hebb (1955), which postulates an inverted-U relationship between performance and arousal, with moderate arousal producing optimal performance.

In conclusion, this study offers some evidence regarding the psychometric characteristics of nonspecific resistance activity and convergent validity regarding the relationships of nonspecific activity to arousal. It is, however, possible that the accuracy of nonspecific activity in its measure of arousal depends upon the situation in which it is utilized. Further investigations under different conditions are necessary, especially those utilizing conditions that will produce a wider range of arousal. In the appropriate experimental setting, this measure might act as a substitute for other more complex measures of arousal, removing some of the methodological restrictions involved with such measures.

\section{REFERENCES}

Hebb, D. O., Drives and the C. N. S. (Conceptual Nervous System). Psychological Review, 1955, 62, 243-253.

Lacey, J. I., \& Lacey, B. C. The relationship of resting autonomic activity to motor impulsivity. In The brain and human behavior. Research Publication of the Association for Research in Nervous and Mental Disease, Vol. XXXVI.

Lykken, D. T., \& Venables, P. H. Direct measurement of skin conductance: A proposal for standardization. Psychophysiology, 1971, 8, 656-672.

McDonald, D. G., Johnson, L. C., \& Hord, D. J. Habituation of the orienting response. Psy chophysiology, 1964, 1, 163-173. Siegel, S. Nonparametric statistics for the behavioral sciences. New York: McGraw-Hill, 1956.

Zuidema, G. P., Cohen, S. F., Silverman, A. J., \& Riley, M. D. Human tolerance to prolonged acceleration. Journal of Aviation Medicine, 1956, 27, 469-481.

(Received for publication May 7, 1975.) 